\title{
Dielectric spectroscopy of palm olein during batch deep frying and their relation with degradation parameters
}

\begin{abstract}
Total polar compounds (TPC) and free fatty acids (FFA) are important indicators in evaluating the quality of frying oil. Conventional methods to determine TPC and FFA are often time consuming, involved laboratory analyses which required skilled personnel and used substantial amount of harmful solvent. In this study, dielectric spectroscopy technique was used to investigate the relation between dielectric property of refined, bleached and deodorized palm olein (RBDPO) during deep frying with TPC and FFA. In total, 150 batches of French fries were intermittently fried at $185 \pm 5^{\circ} \mathrm{C}$ for $7 \mathrm{hr}$ a day over 5 consecutive days. A total of 30 frying oil samples were collected. The dielectric property of frying oil samples were measured using impedance analyzer with frequencies ranging from $100 \mathrm{~Hz}$ to $10 \mathrm{MHz}$. The TPC of frying oil samples were measured with a Testo 270, while the FFA analysis was done using Malaysian Palm Oil Board (MPOB) test method. Results showed that dielectric constant, TPC and FFA of RBDPO increased as the frying time increased. Dielectric constant increased from 3.09 to 3.17, while TPC and FFA increased from 9.96 to 19.52 and from $0.08 \%$ to $0.36 \%$, respectively. Partial least square (PLS) analysis produced good prediction of TPC and FFA with the application of genetic algorithm (GA). Model developed for prediction of TPC and FFA yielded highly significant correlation with R2 of 0.91 and 0.95 , respectively and both had root mean square error in cross-validation (RMSECV) of $1.06 \%$. This study demonstrates the potential of dielectric spectroscopy in monitoring palm olein degradation during frying.
\end{abstract}

Keyword: Dielectric property; Free fatty acids; Palm olein; Total polar compounds 